\title{
PEA-15 contributes to the clinicopathology and AKT-regulated cisplatin resistance in gastric cancer
}

\author{
XIAN JIANG ${ }^{1}$, CHANGLU ZHANG $^{2}$, WEIDONG LI ${ }^{1}$, DALEI JIANG ${ }^{3}$, ZHENG WEI $^{1}$, \\ MEI LV ${ }^{3}$, XIANGJUN XIE ${ }^{3}$ and XUEYING SUN ${ }^{1}$ \\ ${ }^{1}$ Department of General Surgery, The First Affiliated Hospital of Harbin Medical University, Harbin, Heilongjiang 150001; \\ Departments of ${ }^{2}$ Intensive Care Unit and ${ }^{3}$ Gastroenterology, Qingdao Municipal Hospital, \\ Qingdao, Shandong 266011, P.R. China
}

Received July 11, 2018; Accepted November 29, 2018

DOI: 10.3892/or.2018.6934

\begin{abstract}
Phosphoprotein enriched in astrocytes 15 (PEA-15) plays an important role in controlling biological behaviors of cancer cells. In the present study, we demonstrated that PEA-15 was overexpressed in gastric cancer tissues and associated with tumor staging, differentiation, pathological types and the prognosis of patients. Gastric cancer cells expressed variable levels of PEA-15 and its bi-phosphorylation forms, p-PEA-15 (Ser104) and p-PEA-15 (Ser116). To gain insight into the functional role of PEA-15, we generated cells stably depleted of PEA-15 and resistant to cisplatin (CDDP) from human gastric cancer cells. PEA-15 depletion inhibited cell proliferation by reducing cyclin D1 expression through the extracellular signal-regulated kinase (ERK) pathway, resulting in cell cycle arrest at the G1 phase, and induced apoptosis by activating caspase-8. PEA-15 depletion also enhanced the inhibitory effect of CDDP that caused cell cycle arrest at the S phase and also enhanced the pro-apoptotic activity of CDDP in vitro and in animal models of tumorigenesis and therapeutic effects. PEA-15 and its phosphorylated forms were overexpressed in CDDP-resistant cells, which had higher levels of p-AKT. Specific inhibition of AKT by MK2206 reduced the expression of p-PEA-15 at the Ser116 residue, resulting in sequential downregulation of p-ERK1/2, cyclin D1 and caspase- 8 activation. However, depletion of PEA-15 had little effect on AKT expression or phosphorylation, or its downstream factors
\end{abstract}

Correspondence to: Professor Xueying Sun, Department of General Surgery, The First Affiliated Hospital of Harbin Medical University, 23 Youzheng Street, Harbin, Shandong 150001, P.R. China

E-mail: sunxueying@hrbmu.edu.cn

Professor Xiangjun Xie, Department of Gastroenterology, Qingdao Municipal Hospital, 1 Jiaozhou Road, Qingdao, Shandong 266011, P.R. China

E-mail: xiangjunxie2005@126.com

Key words: gastric cancer, phosphoprotein enriched in astrocytes 15, cisplatin, AKT, extracellular signal-regulated protein kinase including p27, glycogen synthase kinase $3 \beta$ and caspase-9, indicating that the regulatory effects between PEA-15 and AKT were unidirectional. In summary, the results indicated that PEA-15 expression was associated with clinicopathology and prognosis in gastric cancer and was regulated by AKT to participate in CDDP resistance, indicating that it may be a potential target for overcoming CDDP resistance in the treatment of gastric cancer.

\section{Introduction}

Globally gastric cancer is ranked fifth in cancer mortality (1). Despite recent progress, most patients are diagnosed at advanced stages and are not eligible for curative surgery, and hence chemotherapy is essential for their treatment (2). However, conventional chemotherapies display only modest effects on the survival of patients with advanced gastric cancer. Cisplatin (CDDP) is one of the most commonly used chemotherapeutic agents in the clinical management of gastric cancer (2), and included in the first-line regimen for advanced gastric cancer in Japan (3). Unfortunately, resistance to CDDP largely limits its beneficial effects, representing a major obstacle to effective treatments. Therefore, exploring the mechanisms for this resistance is required for devising therapeutic strategies to combat gastric cancer.

Phosphoprotein enriched in astrocytes 15 (PEA-15), also known as phosphoprotein enriched in diabetes (PED), is ubiquitously expressed in mammals and was originally identified in primary cultured astrocytes (4). PEA-15 was initially found to play a prominent role in controlling cell survival and glucose metabolism (5), and later it was demonstrated to participate in regulating multiple biological behaviors of cells from several types of malignancies including breast and colorectal cancer, and hepatocellular carcinoma (HCC) (6-9). The well-known mechanism accounting for its function is the regulation of the activation of extracellular signal-regulated protein kinases 1 and 2 (ERK1/2), which mediate cell proliferation, migration and apoptosis $(6,10)$. However, its role in gastric cancer remains unknown. Therefore, the present study was designed to examine the expression of PEA-15 in human gastric cancer tissues and investigate its functional role in gastric cancer cells. 
In addition, PEA-15 has recently been demonstrated to contribute to the insensitivity of a panel of chemotherapeutic agents including CDDP in breast cancer (11), resistance to fluorouracil and CDDP in colon cancer (8), and sorafenib resistance in HCC (9). However, it is unclear whether PEA-15 contributes to the mechanisms of CDDP resistance in gastric cancer. We have previously reported that CDDP-resistant gastric cancer cells expressed higher levels of phosphorylated AKT (p-AKT) (12). PEA-15 features an AKT phosphorylation motif upstream from Ser116 and the phosphorylation by AKT affects its antiapoptotic function $(11,13,14)$. Therefore, we hypothesized that PEA-15 may be involved in AKT activation-regulated mechanisms of CDDP resistance in gastric cancer cells.

\section{Materials and methods}

Patients. The general information of the patients used in the present study has been previously described (15). Briefly, a total of 141 consecutive patients with gastric cancer received surgical treatments at Qingdao Municipal Hospital in China. The diagnosis of gastric cancer was pathologically confirmed and histological classification was performed according to the 2010 World Health Organization (WHO) histological classification system and cell differentiation (16). The disease was staged in accordance with the staging system of the American Joint Committee on Cancer (16). None of the patients had received any preoperative anticancer treatments. The study analyzing human specimens was approved by the Ethics Committee of Qingdao Municipal Hospital (no. 20150819), and informed consents were obtained (Qingdao, China). The animal experiments were approved (permit no. SYXK20020009) by the Animal Ethics Committee of Harbin Medical University (Harbin, China).

Cell culture, antibodies and reagents. Human gastric cancer cells MGC-803, SGC7901, BGC823, AGS, NCI-N87 and HGC-27 were obtained from the Type Culture Collection Cell Bank (Chinese Academy of Sciences Committee, Shanghai, China). Cells were cultured in RPMI-1640 medium supplemented with $10 \%$ fetal bovine serum at $37^{\circ} \mathrm{C}$ in a humidified atmosphere of $5 \% \mathrm{CO}_{2}$. All cell lines were confirmed negative for mycoplasma infection using a PCR-based Universal Mycoplasma Detection kit (American Type Culture Collection, Manassas, VA, USA). An antibody (Ab) against PEA-15 (SAB4503451), FASTDAB (3,3'-diaminobenzidine tetrahydrochloride) and $\mathrm{CoCl}_{2}$ enhancer tablets, and a TUNEL (Terminal deoxynucleotidyl transferase-mediated dUTP nick end labeling agent) kit were purchased from Sigma-Aldrich (Shanghai, China). An anti-PEA-15 (Ser116) Ab (cat. no. PA5-38314) was purchased from Thermo Fisher Scientific Inc. (Shanghai, China). Abs against p-PEA-15 (Ser104) (cat. no. 2776), ERK1/2 (cat. no. 4695), p-ERK1/2 (Thr202/Thyr204) (cat. no. 4370), AKT (cat. no. 4691), p-AKT (Ser473) (cat. no. 4060), caspase-3 (cat. no. 9662), p27 (cat. no. 3688), cyclin D1 (cat. no. 2922), GSK-3 (cat. no. 9315), p-GSK-3 (cat. no. 5558) and caspase-9 (cat. no. 9508) were provided by Cell Signaling Technology (Boston, MA, USA). Abs against $\beta$-actin (cat. no. sc-130065), caspase-9 (cat. no. sc-56073), caspase-8 (cat. no. sc-56070), caspase- 8 (cat. no. sc-73526) were obtained from Santa Cruz Biotechnology (Santa Cruz, CA, USA). An anti-Ki-67 Ab (cat. no. ab15580) and a caspase-3 activity kit (cat. no. ab39401) were purchased from Abcam (Shanghai, China).

Immunohistochemical analysis of human specimens. The methods have been previously described (15). Briefly, tissues collected from patients during surgery were formalin-fixed, paraffin-embedded, sectioned and mounted on 3-aminopropyltriethoxysilane-coated slides. After antigen retrieval, the sections were blocked and incubated with a rabbit anti-human PEA-15 Ab (diluted at 1:250) at $4^{\circ} \mathrm{C}$ overnight. A standard horseradish peroxidase staining procedure was performed using a biotinylated secondary $\mathrm{Ab}$ (diluted at 1:250), and immunoreactivity developed with Sigma FAST DAB and $\mathrm{CoCl}_{2}$ enhancer tablets. Normal rabbit sera (diluted at $1: 10$ ) were used for blocking and dilution of Abs. Negative controls were achieved using irrelevant goat IgG (diluted at 1:50). Immunostaining was assessed in 20 randomly selected fields per specimen using a semi-quantitative grading system. The staining intensity (Value A) was graded in a four-tier grading system: No staining (0), faint yellow (1), yellow (2) and brown (3); while the extent of positive staining (Value B) was graded in a four-tier grading system based on the percentage of positive cells: $\leq 10 \%$ (1), $11-40 \%$ (2), $41-70 \%$ (3) and $\geq 70 \%$ (4). The immunohistological score was calculated by $\mathrm{A} \times \mathrm{B}$, and each specimen was graded lower $(\leq 5)$ or higher level $(>5)$.

Quantitative reverse-transcription polymerase chain reaction ( $q R T-P C R)$. Total RNA was extracted from the cells, and cDNA was synthesized. The reaction mixtures for qRT-PCR were prepared with the primers for PEA-15 (forward, 5'-AGG AAGACATCCCCAGCGAA-3' and reverse, 5'-CCATAGTGA GTAGGTCAGGACG-3'); and $\beta$-actin (forward, 5'-TTAGCA CCCCTGGCCAAGG-3' and reverse, 5'-CTTACTCCTTGG AGGCCATG-3') as previously described (17). Reaction solutions were analyzed by MX3000P Real-Time PCR Systems (Stratagene; Agilent Technologies, Inc., North Billerica, MA, USA). Experiments were performed in triplicate, and the data were calculated using the $\Delta \Delta \mathrm{Cq}$ method (18).

shRNA expression vectors. The previously reported sequences were selected to specifically target PEA-15 (5'-GCGAAAAGA GUGAGGAGAU-3' and 5'-AUCUCCUCACUCUUUUCG C-3') corresponding to nucleotides 611-629 of human PEA-15 (GenBank NM_001297576.1) as previously reported (19). The oligonucleotides were introduced into the pSuppressorNeo vector to generate PEA-shRNA. A scrambled shRNA vector (Sc-shRNA) targeting non-specific sequences (5'-GAAGAC GAAGAGUGAGGAU-3' and 5'-AUCUCCACUCUCUGU UCUC-3') served as a control.

Animal experiments. A total of 46 male nude BALB/c mice (H-2b) (aged 6-8 weeks; body weight, 20.3 $\pm 2.5 \mathrm{~g}$ ) were obtained from the Animal Research Center, The First Affiliated Hospital of Harbin Medical University (Harbin, China). Animals were housed in cages under pathogen-free conditions at a temperature of $20-25^{\circ} \mathrm{C}$ and 12 -h light/dark cycle, and fed commercial pellets and water ad libitum. The experimental protocol has been previously described $(12,15,20)$. Two sets of experiments were designed for evaluating the functional role of PEA-15 in gastric cancer cells in vivo. 
Table I. Association between PEA-15 expression and clinicopathology of gastric cancer.

\begin{tabular}{|c|c|c|c|c|}
\hline & $\mathrm{n}$ & Lower level of PEA-15 & Higher level of PEA-15 & P-value \\
\hline Sex & & & & 0.355 \\
\hline Male & 87 & 38 & 49 & \\
\hline Female & 54 & 20 & 34 & \\
\hline $\begin{array}{l}\text { Age median (range): } \\
53.4(27-82)\end{array}$ & & & & 0.387 \\
\hline$<60$ & 93 & 32 & 61 & \\
\hline$\leq 60$ & 48 & 21 & 27 & \\
\hline TNM stage & & & & 0.011 \\
\hline 0 & 5 & 5 & 0 & \\
\hline I & 26 & 18 & 8 & \\
\hline II & 38 & 25 & 13 & \\
\hline III & 49 & 22 & 37 & \\
\hline IV & 23 & 5 & 18 & \\
\hline WHO histological classification & & & & 0.039 \\
\hline Tubular & 67 & 41 & 26 & \\
\hline Papillary & 42 & 19 & 23 & \\
\hline Mucinous & 13 & 3 & 10 & \\
\hline Poorly cohesive & 11 & 2 & 9 & \\
\hline Uncommon histologic variants & 8 & 4 & 4 & \\
\hline Differentiation & & & & 0.008 \\
\hline Well & 35 & 28 & 7 & \\
\hline Moderate & 74 & 40 & 34 & \\
\hline Poor & 32 & 6 & 26 & \\
\hline
\end{tabular}

P-value was estimated by Mann-Whitney U test. PEA-15, phosphoprotein enriched in astrocytes-15; TNM, tumor-node-metastasis.

Tumorigenicity. Cells $\left(2 \times 10^{6}\right)$ were subcutaneously injected into the flanks of mice, which were monitored to check the appearance of tumors, and tumor size was measured every 4 days. Tumor volumes were calculated according to the following formula: $\pi / 6 \times a^{2} \times b$, where $a$ is the short $a x$ is and $b$ the long axis. Mice were sacrificed 28 days later, and tumors were harvested and weighed.

Therapeutic study. Cells $\left(2 \times 10^{6}\right)$ were subcutaneously injected into the flanks of mice. When tumors reached $\sim 100 \mathrm{~mm}^{3}$, the mice were assigned to 4 groups: Control ( $n=8)$, CDDP $(n=6)$, PEA-shRNA $(n=8)$ and CDDP + PEA-shRNA $(n=6)$. Each mouse in each group received both intratumoral and intraperitoneal injections. Normal saline (200 $\mu \mathrm{l})$ and CDDP $(0.25 \mathrm{mg} / \mathrm{mouse}$, dissolved in $0.9 \%$ sodium chloride $0.9 \%)$ were intraperitoneally injected every 3 days. The shRNA transfection solution was prepared by mixing the shRNA vector, Lipofectamine 2000 and serum-free medium, and was intratumorally injected at a dose of $50 \mathrm{ml}$ containing $200 \mu \mathrm{g}$ shRNA. The doses have been previously reported $(12,15)$. Sc-shRNA was administered in the control and CDDP groups; saline, in the control and PEA-shRNA groups; CDDP, in the CDDP and CDDP + PEA-shRNA groups; and PEA-shRNA, in the PEA-shRNA and CDDP + PEA-shRNA groups. Four days after commencement of treatments, 2 mice from the control and PEA-shRNA groups each were sacrificed to examine tumoral expression of PEA-15. The other mice were monitored and euthanized by carbon dioxide exposure on day 20 .

Establishment of stable transfectants, cell viability analysis, bromodeoxyuridine incorporation proliferation assay, cell cycle assessment, transfection of siRNA, CDDP-resistant cells, immunoblotting, immunohistochemistry, in situ Ki-67 proliferation and in situ cell death detection. The detailed methods have been previously described (21-23), and are available upon request.

Statistical analysis. The association between the expression of PEA-15 and clinicopathology of gastric cancer was analyzed using a Mann-Whitney U test. The Kaplan-Meier method was used to estimate the association between the expression levels of PEA-15 and the survival of patients, and a log-rank test was used to compare the survival curves of 2 groups of patients with low (grade $\leq 5$ ) and high (grade $>5$ ) expression levels of PEA-15. Other data were expressed as the mean values \pm standard deviation, and statistical analysis was performed using a one-way analysis of variance (ANOVA) followed by Dunnett's test. $\mathrm{P}<0.05$ was considered to indicate a statistically significant result. 

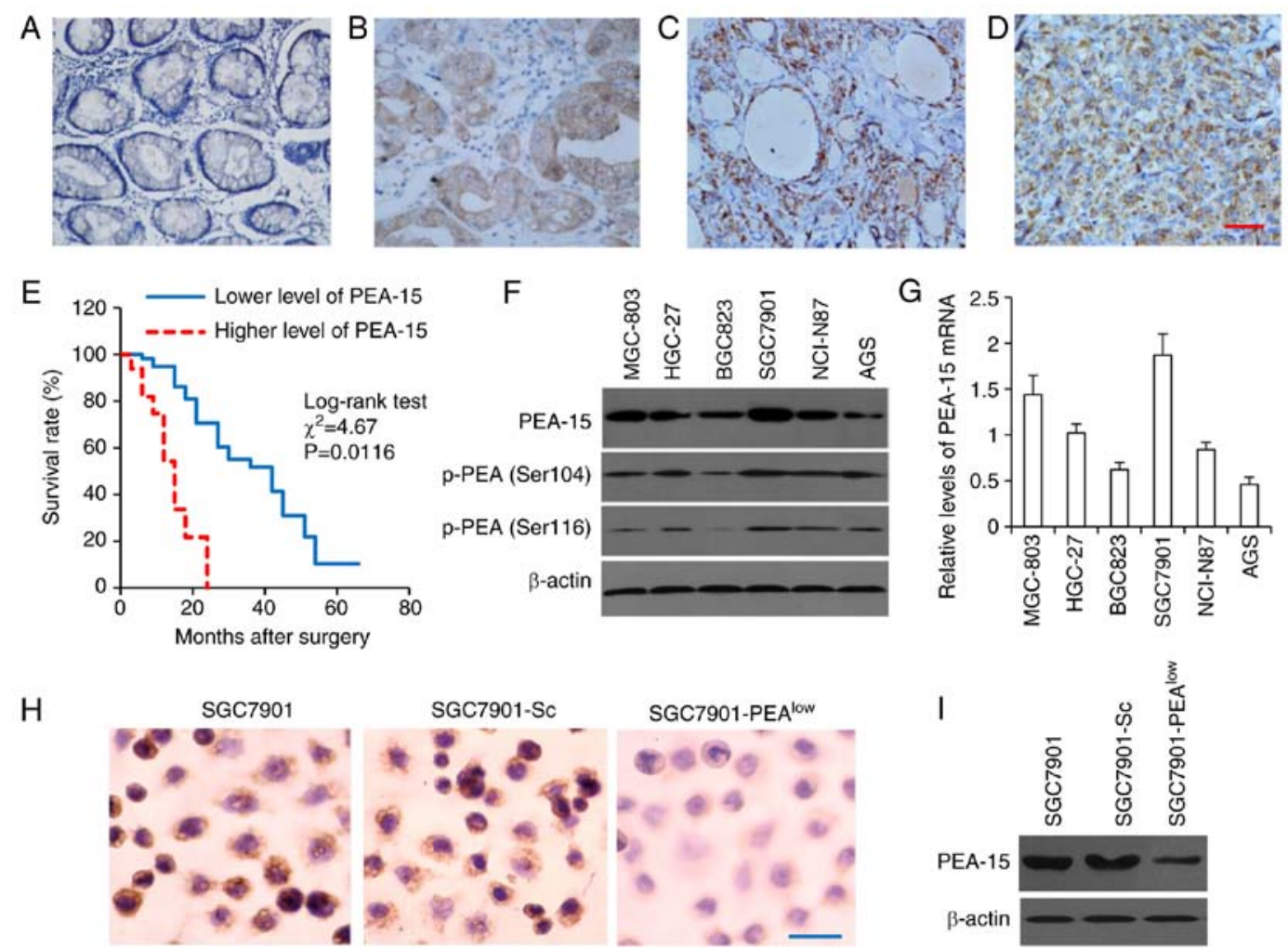

Figure 1. The expression of PEA-15 in gastric cancer tissues and cells. (A-D) Representative images are sections from (A) gastric normal mucosa, (B) adenocarcinoma, (C) mucinous and (D) undifferentiated cancer, immunostained with an anti-PEA-15 antibody (magnification bar, $50 \mu \mathrm{m}$ ). (E) The association between PEA-15 expression and the overall survival was assessed, and a log-rank test was used to analyze the survival curves of patients with lower and higher expression levels of PEA-15. (F and G) The expression of PEA-15 (F) protein and (G) mRNA was detected in a panel of human gastric cancer cells by immunoblotting and qRT-PCR, respectively. (H and I) The expression of PEA-15 was examined in parental SGC7901, SGC7901-Sc and SGC7901-PEA ${ }^{\text {low }}$ cells by $(\mathrm{H})$ immunocytochemistry (magnification bar, $10 \mu \mathrm{m}$ ) and (I) immunoblotting. PEA-15, phosphoprotein enriched in astrocytes 15.

\section{Results}

PEA-15 expression is associated with the clinicopathology and prognosis of gastric cancer. The expression of PEA-15 in tissues was examined using immunohistochemistry, which revealed that normal gastric mucosa had weaker PEA-15 expression, while gastric cancer tissues expressed higher levels of PEA-15 (Fig. 1 A-D), although the expression levels varied (Table I). The results of Mann-Whitney analysis revealed that the expression levels of PEA-15 were not significantly associated with the sex or age of the patients, but significantly associated with TNM staging, tumor differentiation and pathological types according to the criteria of WHO histological classification (Table I). These results indicated that high PEA-15 expression may be associated with advanced stages and poor differentiation of gastric tumors. In addition, through a follow-up of patients after surgery, Kaplan-Meier survival curves were used to analyze the association between the expression levels of PEA-15 and the prognosis of patients. We found that patients with lower PEA-15 expression had a significantly longer overall survival time (median 42.5 months) than those with higher PEA-15 expression (median 13.7 months) (Fig. 1E), indicating that higher PEA-15 expression may be associated with a poor prognosis in gastric cancer.

PEA-15 expression in gastric cancer cell lines. To gain insight into the functional role of PEA-15 in gastric cancer, we examined
PEA-15 expression in a panel of available human gastric cancer cell lines. PEA-15 protein expression was variable among these cell lines, thus SGC7901 and MGC-803 cells expressed higher levels of PEA-15, while BGC823 and AGS, lower levels of PEA-15 (Fig. 1F). Notably, the bi-phosphorylation forms of PEA-15, p-PEA-15 (Ser104) and p-PEA-15 (Ser116) were also observed (Fig. 1F). In addition, we assessed PEA-15 mRNA expression by qRT-PCR, which revealed a similar variability to that of protein expression (Fig. 1G). Since SGC7901 cells were revealed to express the highest level of PEA-15 among the 6 types of gastric cells, they were selected for generating stable transfectants. SGC7901 cells stably transfected with PEA-15 shRNA or Sc-shRNA were termed SGC7901-PEA ${ }^{\text {low }}$ and SGC7901-Sc, respectively. Compared with the parental SGC7901 cells, SGC7901-PEA ${ }^{\text {low }}$ expressed significantly lower, while SGC7901-Sc cells expressed similar, levels of PEA-15, when examined by immunocytochemistry (Fig. 1H) and immunoblotting (Fig. 1I).

PEA-15 depletion increases the sensitivity of gastric cancer cells to CDDP by inducing cell cycle arrest at the G1 phase. We first demonstrated that PEA-15 depletion inhibited the growth of gastric cancer cells in culture. As revealed in Fig. 2A, SGC7901-PEA ${ }^{\text {low }}$ cells had a significantly lower viability, while SGC7901-Sc cells had a similar viability, compared with parental cells. CDDP treatment reduced the viability of the 3 cell lines in a concentration-dependent manner (Fig. 2B). SGC7901-PEA ${ }^{\text {low }}$ cells were more sensitive to CDDP than 

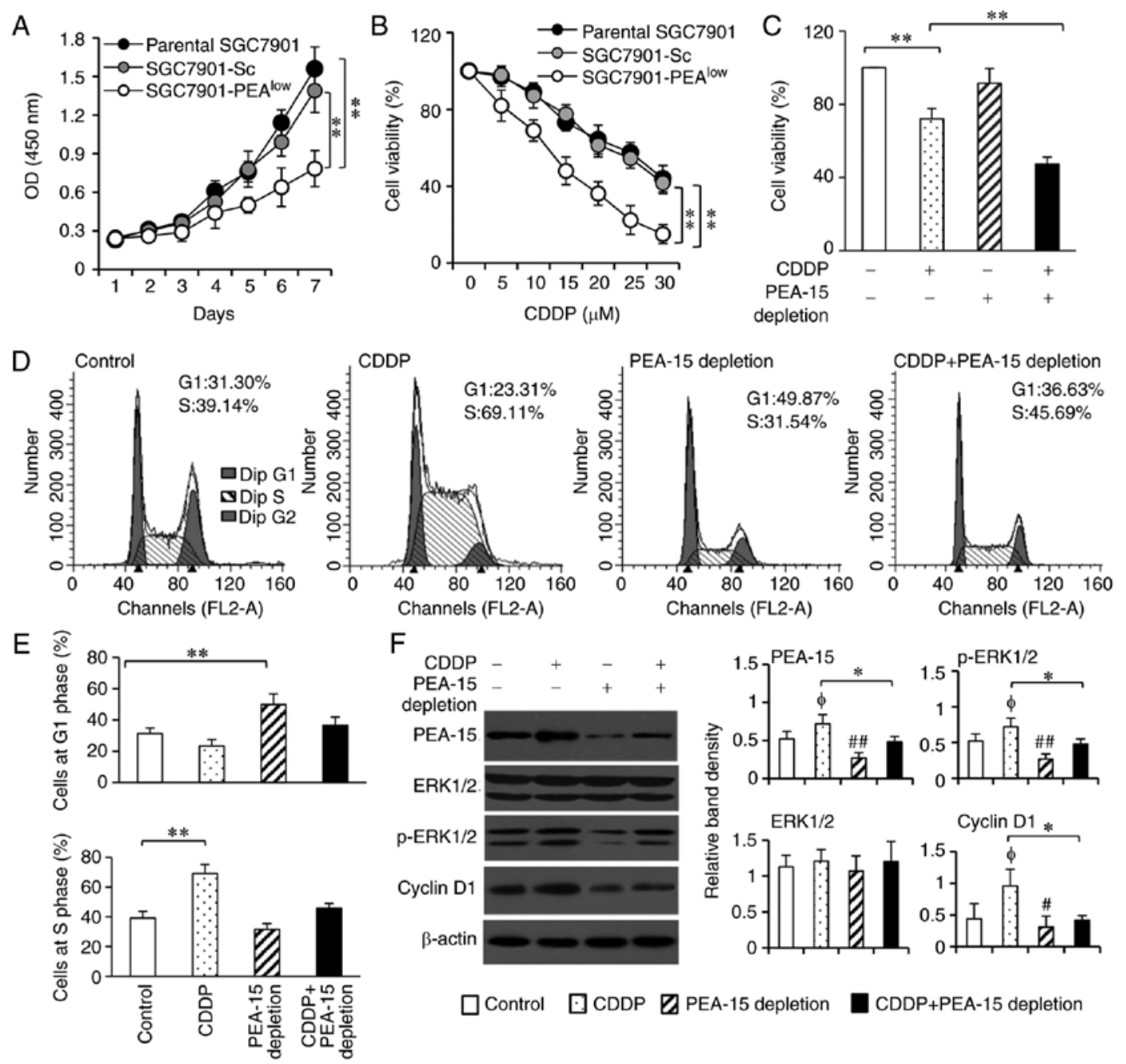

Figure 2. PEA-15 depletion enhances the sensitivity of gastric cancer cells to CDDP by inducing cell cycle arrest at phase G1 through the ERK pathway. (A) Parental SGC7901, SGC7901-Sc and SGC7901-PEA ${ }^{\text {low }}$ cells were cultured for 7 days, and their viability was assessed by a CCK-8 assay at the indicated time-points. Cell viability was represented by optical density (OD) at $450 \mathrm{~nm}$. (B) Cells were incubated with increasing concentrations of CDDP for $48 \mathrm{~h}$, and their percentage viability was compared to that of untreated respective cells. (C) Cells were cultured in the presence or absence of CDDP (15 $\mu \mathrm{M})$ for $48 \mathrm{~h}$, and their viability was assessed. (D and E) The aforementioned cells were subjected to flow cytometry to assess cell cycle distribution. (D) Representative histograms were shown and (E) the percentages of cells at the G1 and S phases were plotted. (F) Lysates from the aforementioned cells were immunoblotted. The density of each band was measured and normalized to $\beta$-actin. ${ }^{*} \mathrm{P}<0.05$ and ${ }^{* *} \mathrm{P}<0.001$ indicate a significant difference. ${ }^{\phi} \mathrm{P}<0.05$ indicates a significant increase, while ${ }^{\#} \mathrm{P}<0.05$ and ${ }^{\# \#} \mathrm{P}<0.01$, indicate a significant reduction, from the control. PEA-15, phosphoprotein enriched in astrocytes 15 ; CDDP, cisplatin; ERK, extracellular signal-regulated kinase; CCK-8, Cell Counting Kit-8.

parental or SGC7901-Sc cells (Fig. 2B). CDDP treatment significantly reduced the viability of both SGC7901 and SGC7901-PEA $^{\text {low }}$ cells, but the viability of SGC7901-PEA ${ }^{\text {low }}$ cells was significantly lower than that of SGC7901 cells after incubation for $48 \mathrm{~h}$ with CDDP $(15 \mu \mathrm{M})$ (Fig. 2C). SGC7901 and SGC7901-Sc cells had revealed similar cell viabilities when they were incubated in the absence or presence of CDDP (data not shown and available upon request).

Analyses of cell cycle distribution revealed that CDDP treatment induced more cells arrested at the $S$ phase, while PEA-15 depletion induced more cells arrested at the G1 phase, compared with the controls (Fig. 2D and E). The anticancer mechanism of CDDP mainly relies on its activity to inhibit DNA replication, and cells in phase G1 appear to be maximally sensitive to CDDP (24). Thus, unsurprisingly PEA-15 depletion enhanced the sensitivity of SGC7901 cells to CDDP and worked together with CDDP to further reduce cell viability (Fig. 2C).
PEA-15 depletion and CDDP inhibits cell proliferation by regulating $E R K /$ cyclin $D 1$. The results of viability and cell cycle distribution were consistent with cell proliferation as examined by BrdU incorporation assays. CDDP treatment and PE-15 depletion resulted in significant decreases in the percentage of BrdU-positive cells, and CDDP treatment further reduced the percentage of BrdU-positive SGC7901-PEA ${ }^{\text {low }}$ cells, compared with the controls (data not shown and available upon request). Previous studies have revealed that PEA-15 promotes cell proliferation by regulating ERK phosphorylation $(9,25)$. As revealed in Fig. 2F, exposure of CDDP significantly increased the expression of p-ERK1/2 though it had no effect on total ERK1/2, resulting in a sequential upregulation of cyclin D1, which is a well-known downstream factor of ERK1/2 and plays key roles in cell cycle transition from the G1 to S phase (26). PEA-15 depletion downregulated the expression of p-ERK1/2 and cyclin D1 expression and could reverse the increased expression of p-ERK1/2 and cyclin D1 induced by CDDP, 
A

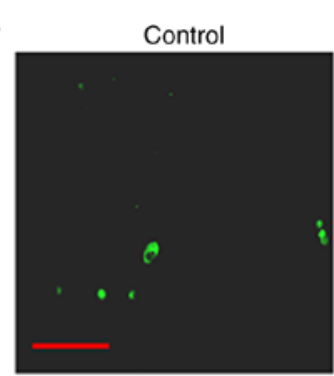

B

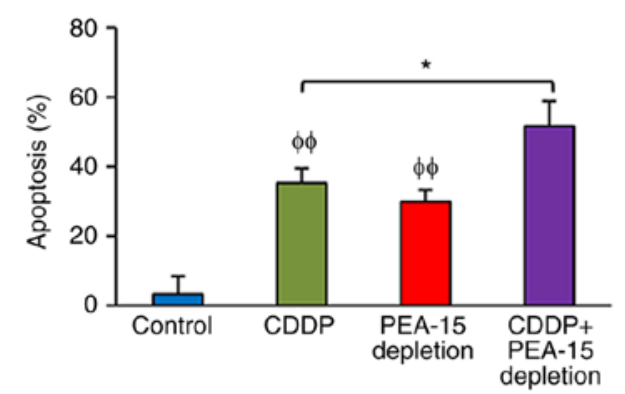

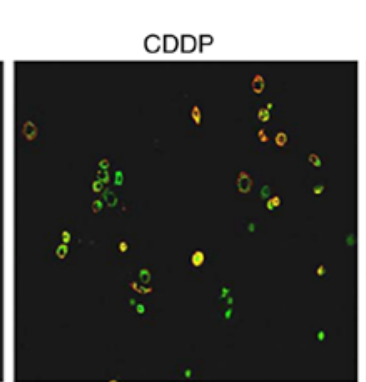

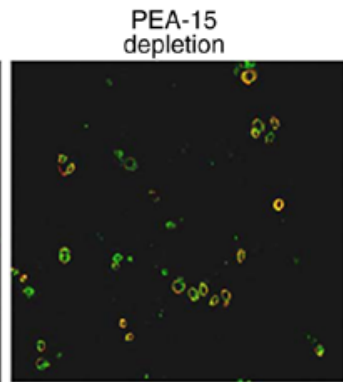

C
CDDP+ PEA-15 depletion
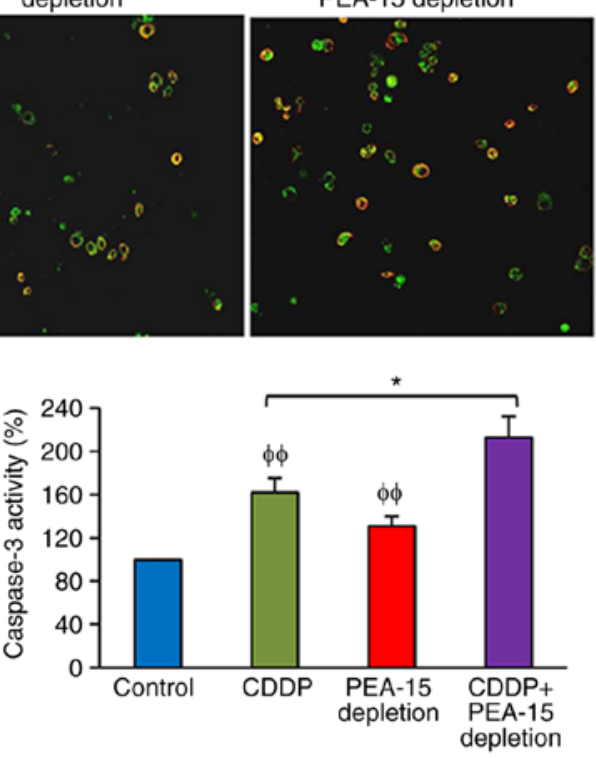

D
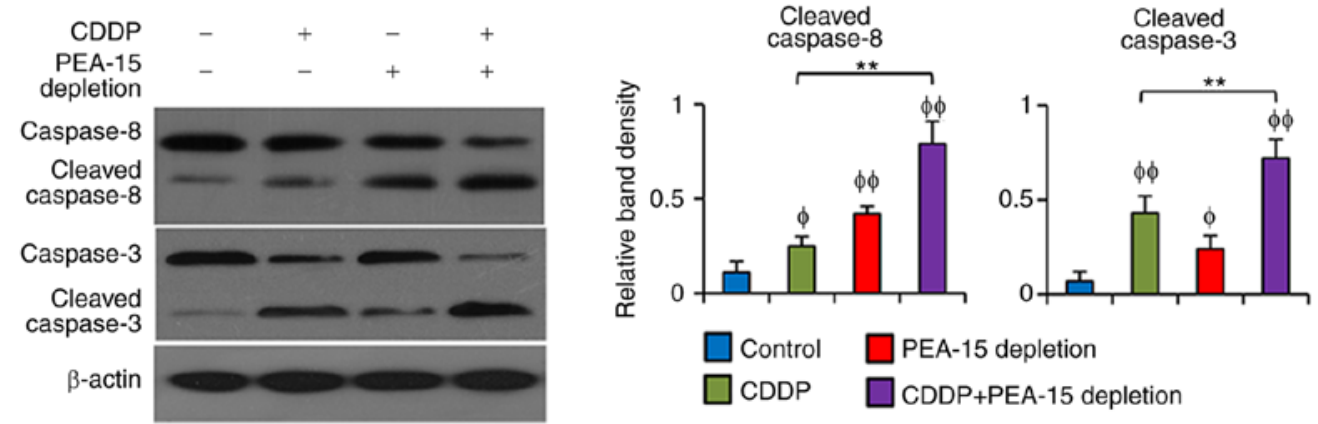

Figure 3. PEA-15 depletion enhances the pro-apoptotic activity of CDDP. SGC7901 and SGC7901-PEA ${ }^{\text {low }}$ cells were cultured in the presence or absence of CDDP $(15 \mu \mathrm{M})$ for $48 \mathrm{~h}$ and subjected to apoptosis assays. (A) Representative images were obtained from cells stained with Annexin V/PI and visualized under laser scanning confocal microscopy. Magnification bar, $25 \mathrm{~mm}$. (B) Apoptosis rates (\%) were measured by flow cytometry. (C) Cells were lysed and caspase-3 activity was assessed. (D) Cell lysates were immunoblotted. The density of each band was measured and normalized to $\beta$-actin. ${ }^{*} \mathrm{P}<0.05$ and ${ }^{* *} \mathrm{P}<0.001$ indicate a significant difference. ${ }^{\phi} \mathrm{P}<0.05$ and ${ }^{\phi \phi} \mathrm{P}<0.001$ indicate a significant increase from control. PEA-15, phosphoprotein enriched in astrocytes 15 ; CDDP, cisplatin.

in SGC7901-PEA ${ }^{\text {low }}$ cells (Fig. 2F). Notably, SGC7901 and SGC7901-Sc cells revealed similar alterations of gene expression upon CDDP incubation (data not shown and available upon request). The aforementioned results indicated that PEA-15 depletion enhanced the sensitivity of gastric cancer cells to CDDP and were confirmed using another cell line MGC-803 (data not shown and available upon request).

PEA-15 depletion enhances the pro-apoptotic activity of CDDP in gastric cancer cells. CDDP and PEA-15 depletion significantly increased apoptosis of SGC7901 cells, and CDDP treatment further increased the apoptosis rate of SGC7901-PEA ${ }^{\text {low }}$ cells (Fig. 3A and B). In accordance, both CDDP and PEA-15 increased caspase-3 activity in SGC7901 cells, and CDDP further increased caspase-3 activity in SGC7901-PEA ${ }^{\text {low }}$ cells (Fig. 3C). CDDP had little effect on caspase-8, but significantly increased the cleavage of caspase-3, while PEA-15 depletion increased the cleavage of caspase- 8 and caspase-3, and CDDP treatments further elevated the cleavage of caspase-3 in SGC7901-PEA ${ }^{\text {low }}$ cells (Fig. 3D).

PEA-15 depletion enhances the therapeutic effects of CDDP in gastric cancer animal models. The aforementioned results led us to investigate the function of PEA-1 in vivo. The study of tumorigenesis revealed that tumors were observed in all the 8 mice receiving subcutaneous injection of SGC7901 cells, but in only 5 out of 8 mice receiving SGC7901-PEA ${ }^{\text {low }}$ cells (Fig. 4A). Furthermore, SGC7901-PEA ${ }^{\text {low }}$ tumors grew to $782.3 \pm 94.5 \mathrm{~mm}^{3}(736.4 \pm 138.6 \mathrm{mg}$ in weight), significantly smaller than SGC7901 tumors whose size was $1,813.6 \pm 114.7 \mathrm{~mm}^{3}(1,524 \pm 187.8 \mathrm{mg}$ in weight), 4 weeks after cell inoculation (Fig. 4A and B). The tumors harvested in Fig. 4B were subjected to immunohistochemistry to examine PEA-15 expression, cell proliferation and apoptosis in situ. Consistent with the in vitro results (Fig. 1F and G), SGC7901-PEA ${ }^{\text {low }}$ tumors expressed markedly lower levels of PEA-1 protein than SGC7901 tumors (Fig. 4C). PEA-1 depletion significantly inhibited cell proliferation and promoted apoptosis (Fig. 4C-E). The expression of key molecules in vivo was consistent with that obtained in vitro; and notably p-PEA-15 at both Ser104 and Ser116 residues was also downregulated in SGC7901-PEA ${ }^{\text {low }}$ tumors (data not shown and available upon request).

In the present study, to assess the therapeutic effects, we established subcutaneous tumors using another gastric cancer cell line, MGC-803, which was also revealed to express 
A

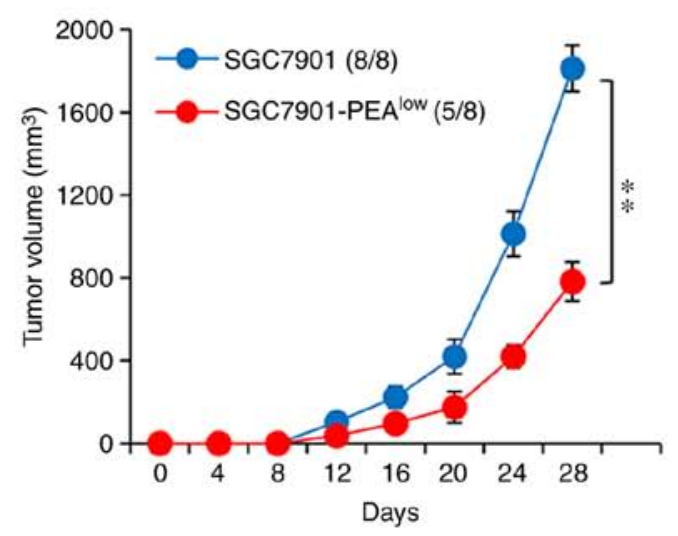

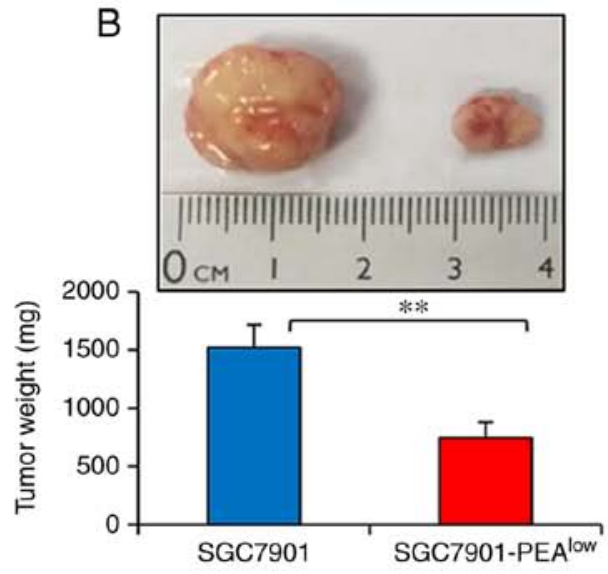

TUNEL

C Anti-PEA-15
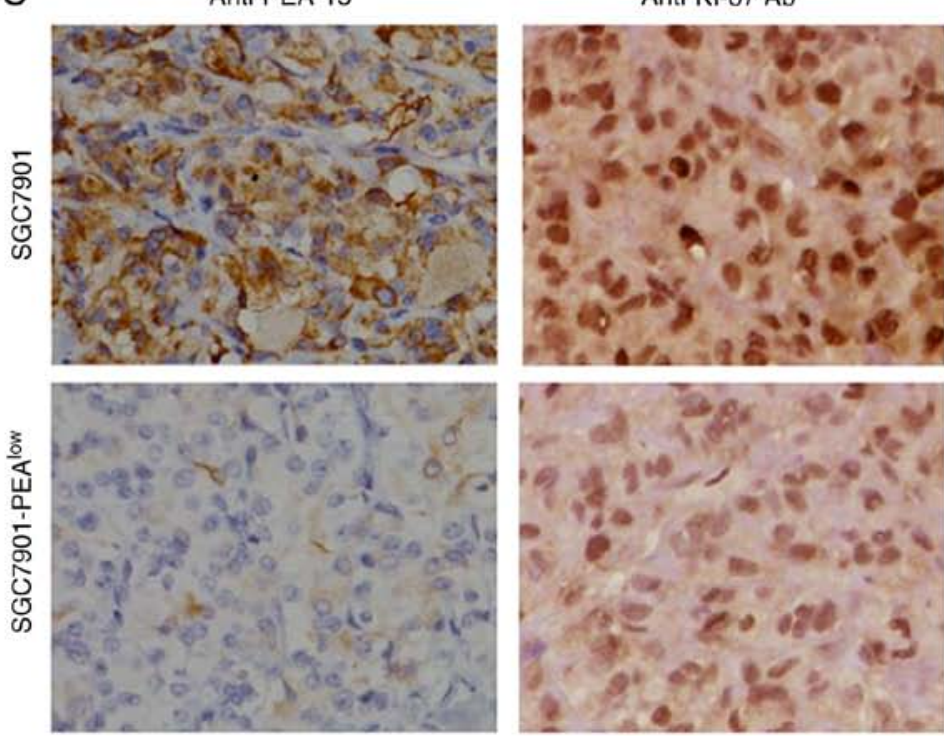

D
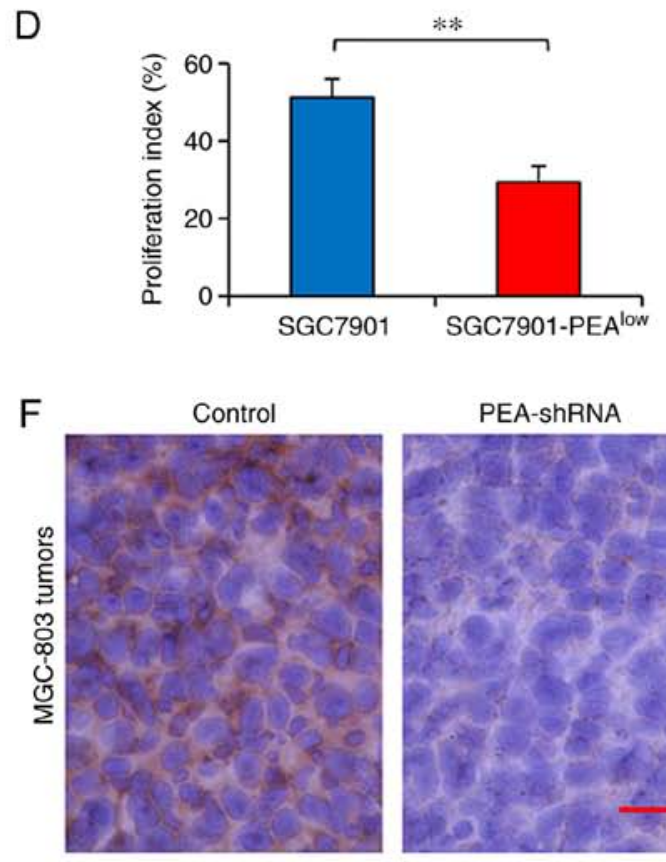

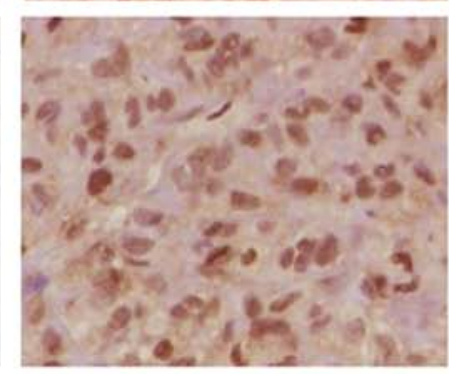

E
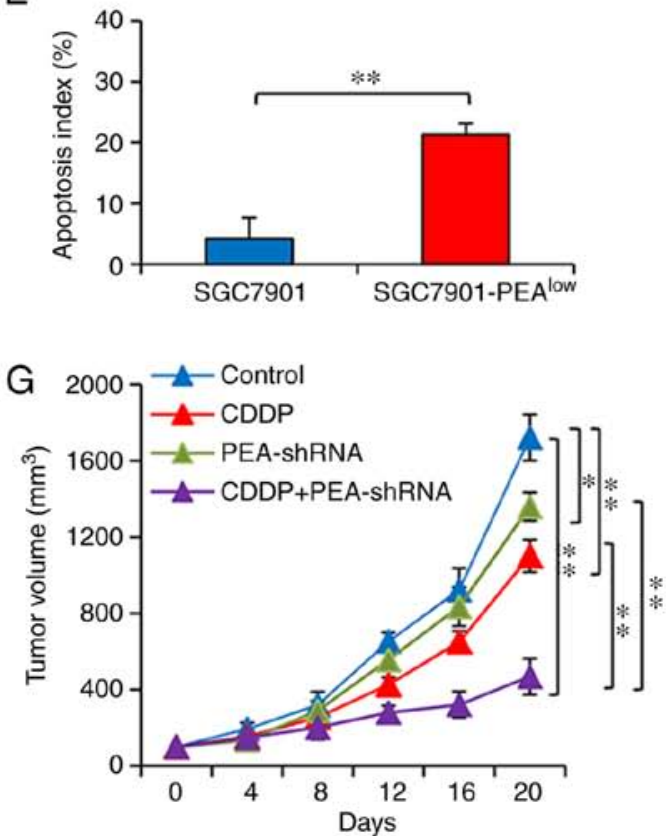

Figure 4. Animal experiments. (A) SGC7901 and SGC7901-PEA ${ }^{\text {low }}$ cells were inoculated into mice. The appearance of palpable tumors was monitored, and the size of tumors were assessed. (B) Tumors were harvested and weighed. (C) Representative images were from tumor sections obtained from B and immunostained as indicated. (D) Tumor in situ proliferation index and (E) apoptosis index were quantified. (F and G) MGC-803 tumors were established in mice, which were assigned to different treatment groups. (F) Representative images of MGC-803 tumor sections immunostained with an anti-PEA-15 Ab. These tumors were harvested on day 4 after treatments started in G. (G) The size of tumors was assessed. Magnification bar, $10 \mu \mathrm{m}$. ${ }^{*} \mathrm{P}<0.05$ and ${ }^{* *} \mathrm{P}<0.001$ indicate a significant difference. PEA-15, phosphoprotein enriched in astrocytes 15. 
A

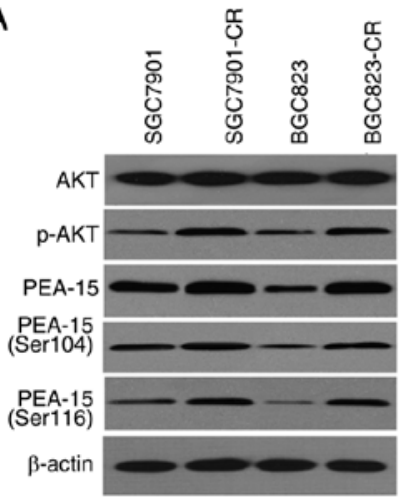

C

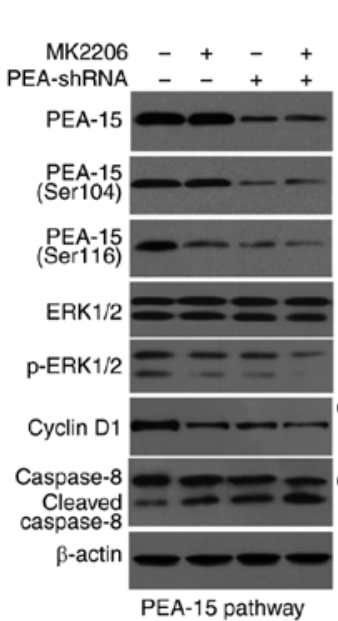

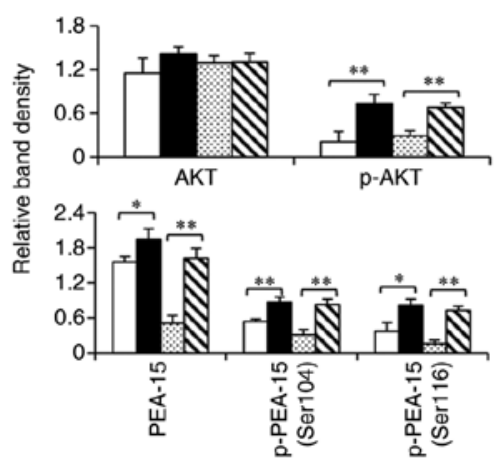

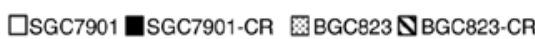

B

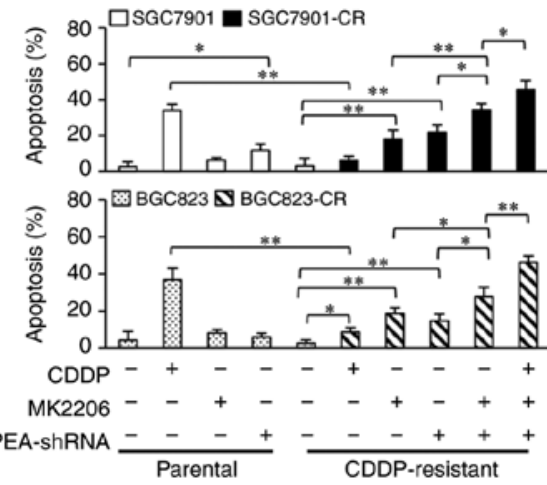

Figure 5. PEA-15 and AKT participate in the mechanisms of CDDP resistance. (A) SGC7901, SGC7901-CR, BGC823 and BGC823-CR cells were immunoblotted. (B) The aforementioned cells were transfected with or without PEA-shRNA, and $24 \mathrm{~h}$ later incubated in the presence or absence of CDDP (15 $\mu \mathrm{M})$, and/or MK2206 $(1 \mu \mathrm{M})$ for $48 \mathrm{~h}$. Cells were subjected to flow cytometry to assess the apoptosis rate (\%). (C) Untransfected and SGC7901 cells transfected with PEA-shRNA were incubated in the presence or absence of MK2206 $(1 \mu \mathrm{M})$ for $48 \mathrm{~h}$. Cell lysates were immunoblotted. The density of each band was measured and normalized to $\beta$-actin. ${ }^{*} \mathrm{P}<0.05$ and ${ }^{* *} \mathrm{P}<0.001$ indicate a significant difference. $\mathrm{PEA}-15$, phosphoprotein enriched in astrocytes 15 ; $\mathrm{CDDP}$, cisplatin.

higher levels of PEA-15 (Fig. 1F and G). When tumors reached $\sim 100 \mathrm{~mm}^{3}$, they were assigned to different treatments as indicated in Materials and methods. Intratumoral delivery of PEA-shRNA resulted in downregulation of PEA-1 in situ, compared with control tumors injected with Sc-shRNA (Fig. 4F). MGC-803 tumors injected with PEA-shRNA were significantly smaller $\left(1,360.4 \pm 75.3 \mathrm{~mm}^{3}\right)$ than control tumors $\left(1719.5 \pm 123.1 \mathrm{~mm}^{3}\right), 20$ days after the commencement of treatments (Fig. 4G). CDDP treatment also led to a significant reduction in the size of tumors injected with Sc-shRNA $\left(1,098.6 \pm 123.1 \mathrm{~mm}^{3}\right)$ and further reduced the size of tumors injected with PEA-shRNA $\left(467.9 \pm 88.7 \mathrm{~mm}^{3}\right)$ (Fig. 4G).

PEA-15 regulated by AKT participates in the mechanisms for CDDP resistance of gastric cancer cells. We previously reported that activation of AKT contributes to the resistance of gastric cancer cells to CDDP (12), and AKT is an upstream factor of PEA-15 by regulating its phosphorylation $(11,13,14)$. Therefore, the functional role of PEA-15 in the mechanisms of CDDP resistance was investigated using SGC7901 cells expressing higher levels of PEA-15, BGC823 cells expressing lower levels of PEA-15, and 2 CDDP-resistant cell lines (SGC7901-CR and BGC823-CR) that were previously established (12). As anticipated, CDDP-resistant cells expressed a similar level of AKT but an increased level of p-AKT, compared with respective parental cells (Fig. 5A), in accordance with our previous study (12). CDDP-resistant cells had higher expression of PEA-15 than their respective parental cells, but the difference was less pronounced for SGC7901 cells which had a high baseline of PEA-15 expression (Fig. 5A). Notably, the expression of p-PEA-15 at both Ser116 and Ser104 residues was also increased in CDDP-resistant cells (Fig. 5A). In addition, SGC7901-CR and BGC823-CR cells had significantly lower rates of apoptosis, compared with respective parental cells, when they were incubated for $48 \mathrm{~h}$ with CDDP (Fig. 5B). Specific inhibition of AKT by a specific AKT inhibitor (MK2206), a novel selective inhibitor of pan-Akt (27), resulted in significantly higher apoptosis rates of SGC7901-CR and BGC823-CR cells (Fig. 5B). Transfection of PEA-shRNA also significantly increased apoptosis rates of SGC7901-CR and BGC823-CR cells. In addition, combination of MK2206 and PEA-shRNA transfection resulted in higher apoptosis rates of CDDP-resistant cells than either MK220 or PEA-shRNA alone (Fig. 5B). 


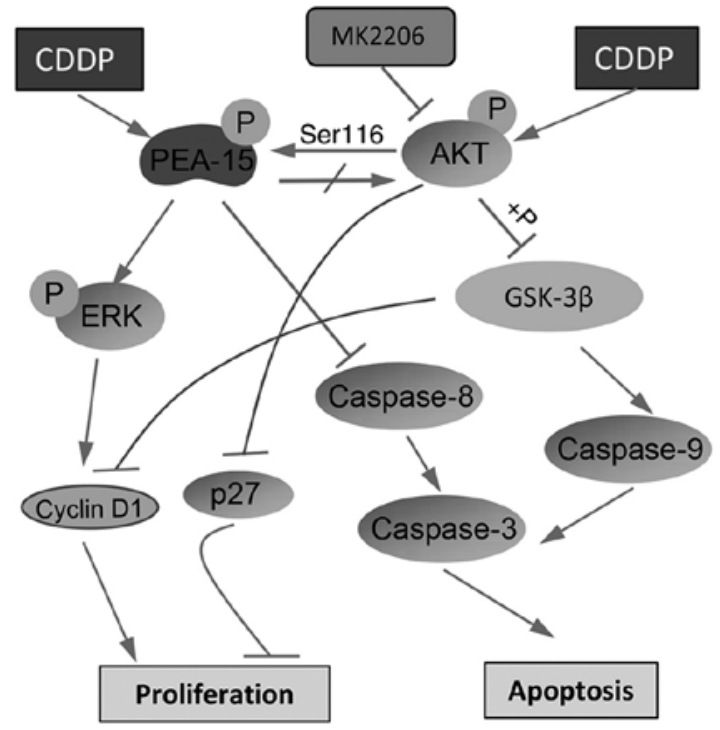

Figure 6. The proposed mechanism of PEA-15 in regulating cell biological behaviors and CDDP resistance of in gastric cancer cells. ' $\rightarrow$ ' indicates promotion, positive regulation or activation. ${ }^{\perp}$ ' indicates inhibition, negative regulation or blockade. PEA-15, phosphoprotein enriched in astrocytes 15; CDDP, cisplatin.

In exploring the regulatory mechanisms between PEA-15 and AKT pathways, SGC7901-Sc and SGC7901-PEA ${ }^{\text {low }}$ cells were incubated with MK2206 and the expression of key molecules involved in the 2 pathways was detected. As revealed in Fig. 5C, MK2206 significantly inhibited the AKT pathway, as evidenced by the reduced expression of p-AKT and its downstream factors including $\mathrm{p} 27$, phosphorylated glycogen synthase kinase $3 \beta$ (p-GSK-3 $\beta$ ), and the increased cleavage of caspase- 9 and -3 . MK2206 had little effect on the expression of PEA-15, p-PEA-15 at Ser104 residue or total ERK1/2 however it could significantly reduce the expression of p-PEA-15 at Ser116 residue, resulting in sequential downregulation of p-ERK1/2, cyclin D1 and increased cleavage of caspase-8 (Fig. 5C). The results indicated that inhibition of AKT could regulate the PEA-15 pathway by reducing the phosphorylation of PEA-15 at Ser116 residue. However, depletion of PEA-15 by PEA-shRNA reduced the expression of PEA-15 and the phosphorylation of PEA-15 at both Ser104 and Ser116 residues but had little effect on either the expression of AKT, or p-AKT, or AKT downstream factors including p27, GSK-3 $\beta$ and the cleavage of caspase-9 (Fig. 5C). The results indicated that depletion of PEA-15 had little effect on the AKT pathway, indicating the regulatory effects between PEA-15 and AKT may be unidirectional in gastric cancer cells.

\section{Discussion}

As a multifunctional phosphoprotein, PEA-15 has displayed an important role in several cancer entities (6-9), but its expression and function have not yet been investigated in gastric cancer. The present study demonstrated that PEA-15 was overexpressed in gastric cancer tissues and cells. In addition, clinical gastric tumors with higher expression of PEA-15 had more advanced TNM stages and poorer cell differentiation, and the patients had a shorter survival time. PEA-15 was also associated with histopathological types of gastric cancer. Similarly, it has been reported that PEA-15 overexpression is observed in breast cancer $(11,28), \mathrm{HCC}(9)$, lung cancer (29), esophageal carcinoma (30) and colorectal cancer (8). In these tumors, PEA-15 acted as a tumor promoter and was associated with poor prognosis. Notably, PEA-15 overexpression has been revealed to be associated with a good prognosis in ovarian cancer, where PEA-15 exists in unphosphorylated status $(31,32)$. The difference of its role in tumor promotion or suppression may depend on its phosphorylation status (9). The present results revealed that gastric cancer cells expressed p-PEA-15 at both Ser104 and Ser116 residues, which had also been revealed in HCC samples (9). Therefore, the results may indicate that PEA-15 acts as a tumor promoter in gastric cancer similar to other types of malignancies excluding ovarian cancer.

It has been demonstrated that p-PEA-15 enhanced cell proliferation by activating the ERK pathway $(9,14,25)$, which regulates cyclin D1, a key molecule controlling cell cycle transition from the G1 to $\mathrm{S}$ phase (26). Similarly, it was revealed herein that PEA-15 depletion resulted in reduced expression of p-ERK1/2, leading to downregulation of cyclin D1 and cell cycle arrest at the G1 phase. CDDP belongs to the alkylating agent family and its major mechanism involves inhibiting DNA replication, thus CDDP exposure induces cell cycle arrest at the $\mathrm{S} / \mathrm{G} 2$ phases. Consistently our results revealed that CDDP incubation induced more cells arrested at the $\mathrm{S}$ phase. It has been reported that cells in phase G1 appear to be the most sensitive to CDDP (24). This may partially explain the mechanism involved in the enhanced activity of CDDP by PEA-15 depletion.

CDDP displays its anticancer activity by inducing apoptosis and caspase-3 activation (33). However, the resistance to CDDP largely limits its therapeutic benefits. PEA-15 has been revealed to contribute to the insensitivity or resistance to various chemotherapeutic agents including CDDP in various types of cancer $(8,9,11)$. In the present study, it was demonstrated that PEA-15 depletion re-sensitized CDDP-resistant gastric cancer cells to CDDP. The analysis exploring the mechanisms revealed that PEA-15 depletion inhibited the cleavage of caspase-8, leading to the inhibition of caspase-3 activation. Supportively it has been revealed that PEA-15 displays its anti-apoptotic activity mainly through its binding to Fas-associated protein with death domain (FADD), which regulates the activation of caspase-8 (9).

The AKT pathway has emerged as a potent molecular target for overcoming acquired resistance to cancer chemotherapy, since AKT is a cancer multidrug resistance locus, by phosphorylating many proteins involved in cancer hallmarks including apoptosis resistance $(34,35)$. We have previously reported that CDDP-resistant gastric cancer cells expressed higher levels of $\mathrm{p}-\mathrm{AKT}$, whose activation regulates the resistance to CDDP (12). Conversely, several studies have indicated that AKT is an upstream factor of PEA-15 by phosphorylating it at the site of Ser116 $(11,13,14)$. In the present study, we further confirmed the overexpression of p-AKT and p-PEA-15 at Ser104 and Ser116 residues in CDDP-resistant gastric cancer cells. Specific inhibition of AKT by MK2206 (27) not only inhibited the AKT pathway, but also downregulated p-PEA-15 at Ser116 and the related downstream factors. 
However, depletion of PEA-15 had little effect on the expression of either total AKT or p-AKT, or the related downstream factors. Combination of AKT inhibition and PEA-15 depletion further increased apoptosis rates of CDDP-resistant gastric cancer cells by activating caspase cascades via both caspase- 8 and -9 . The results may indicate that both PEA-15 and AKT contribute to the mechanisms of CDDP resistance but their regulatory effect is unidirectional.

The proposed mechanisms for its functions in influencing the proliferation and apoptosis of gastric cancer cells, involvement in the mechanisms of CDDP resistance, and regulation by AKT are summarized in Fig. 6. PEA-15 participates in controlling cell proliferation by activating ERK, which regulates the expression of cyclin D1 $(9,14,25,26)$. PEA-15 displays its anti-apoptotic activity by binding to FADD, resulting in the inhibition of caspase- 8 and -3 (9). Exposure to CDDP leads to the higher expression of p-PEA-15 and p-AKT, contributing to the mechanisms of CDDP resistance. Activated AKT regulates GSK-3 $\beta$, which controls cell apoptosis and proliferation by regulating $\mathrm{p} 27$, cyclin D1 and caspase-9 $(23,36)$. Activated AKT is also able to mediate cell proliferation and apoptosis by regulating the PEA-15 pathway.

In summary, PEA-15 is overexpressed in gastric cancer tissues and associated with the clinicopathology and prognosis, and contributes to AKT-regulated CDDP resistance. Although not investigated in the present study, PEA-15 may also participate in the mechanisms for drug resistance of gastric cancer by regulating other pathways. For example, PEA-15 was revealed to regulate autophagy by activating the c-Jun N-terminal kinase (JNK) pathway in glioma cells and autophagy has been revealed to be involved in drug resistance in many types of cancer (19). It is well known that PEA-15 activates ERK1/2, which participates in many biological behaviors and drug resistance of cancer cells $(6,10)$. The initial function of PEA-15 is to mediate energy metabolism (5), which is critical for the therapy resistance of cancer cells (37). The present results indicated that PEA-15 may be a valuable biomarker and potential therapeutic target for gastric cancer, particularly for patients expressing higher levels of PEA-15 and becoming resistant to CDDP.

\section{Acknowledgements}

We thank Dr Shiva Reddy from the University of Auckland (New Zealand) for revising and editing the manuscript.

\section{Funding}

The present study was supported by grants from the National Key Research and Development Program of China (no. 2017YFC1308602), the National Natural Scientific Foundation of China (nos. 81472321 and 81703055), and the Fundamental Research Funds for the Provincial Universities in Heilongjiang Province, China (no. 2017LCZX06).

\section{Availability of data and materials}

The majority of the data generated or analyzed during the present study are included in this published article. Some data are not presented and available upon request.

\section{Authors' contributions}

$\mathrm{XJ}, \mathrm{XX}$ and $\mathrm{XS}$ participated in the conception and study design. XJ and WL performed bench and animal experiments. $\mathrm{CZ}, \mathrm{DJ}, \mathrm{ZW}$ and ML participated in examining the clinical specimens and assisted in bench and animal experiments. XJ and $\mathrm{CZ}$ were involved in the data collection and analyses. $\mathrm{XJ}$ and XS drafted the manuscript. XX revised the manuscript. XX and XS supervised the study. All authors read and approved the manuscript and agree to be accountable for all aspects of the research in ensuring that the accuracy or integrity of any part of the work are appropriately investigated and resolved.

\section{Ethics approval and consent to participate}

The study analyzing human specimens was approved by the Ethics Committee of Qingdao Municipal Hospital (no. 20150819), and informed consents were obtained (Qingdao, China). The animal experiments were approved (permit no. SYXK20020009) by the Animal Ethics Committee of Harbin Medical University (Harbin, China).

\section{Patient consent for publication}

Not applicable.

\section{Competing interests}

The authors state that they have no competing interests.

\section{References}

1. Siegel RL, Miller KD and Jemal A: Cancer Statistics, 2017. CA Cancer J Clin 67: 7-30, 2017.

2. Cats A, Jansen EP, van Grieken NC, Sikorska K, Lind P, Nordsmark M, Meershoek-Klein Kranenbarg E, Boot H, Trip AK, Swellengrebel HA, et al: Chemotherapy versus chemoradiotherapy after surgery and preoperative chemotherapy for resectable gastric cancer (CRITICS): An international, open-label, randomised phase 3 trial. Lancet Oncol 19: 616-628, 2018.

3. Koizumi W, Tanabe S, Azuma M, Ishido K, Nishimura K, Sasaki T, Nakatani K, Higuchi K, Nakayama N and Katada C: Impacts of fluorouracil-metabolizing enzymes on the outcomes of patients treated with S-1 alone or S-1 plus cisplatin for first-line treatment of advanced gastric cancer. Int J Cancer 126: 162-170, 2010.

4. Araujo H, Danziger N, Cordier J, Glowinski J and Chneiweiss H: Characterization of PEA-15, a major substrate for protein kinase C in astrocytes. J Biol Chem 268: 5911-5920, 1993.

5. Fiory F, Formisano P, Perruolo G and Beguinot F: Frontiers: PED/PEA-15, a multifunctional protein controlling cell survival and glucose metabolism. Am J Physiol Endocrinol Metab 297: E592-E601, 2009.

6. Greig FH and Nixon GF: Phosphoprotein enriched in astrocytes (PEA)-15: A potential therapeutic target in multiple disease states. Pharmacol Ther 143: 265-274, 2014.

7. Mohammed HN, Pickard MR and Mourtada-Maarabouni M: The protein phosphatase 4-PEA15 axis regulates the survival of breast cancer cells. Cell Signal 28: 1389-1400, 2016.

8. Funke V, Lehmann-Koch J, Bickeboller M, Benner A Tagscherer KE, Grund K, Pfeifer M, Herpel E, Schirmacher P, Chang-Claude J, et al: The PEA-15/PED protein regulates cellular survival and invasiveness in colorectal carcinomas. Cancer Lett 335: 431-440, 2013.

9. Quintavalle C, Hindupur SK, Quagliata L, Pallante P, Nigro C, Condorelli G, Andersen JB, Tagscherer KE, Roth W, Beguinot F, et al: Phosphoprotein enriched in diabetes (PED/PEA15) promotes migration in hepatocellular carcinoma and confers resistance to sorafenib. Cell Death Dis 8: e3138, 2017. 
10. Buonomo R, Giacco F, Vasaturo A, Caserta S, Guido S, Pagliara V, Garbi C, Mansueto G, Cassese A, Perruolo G, et al: PED/PEA-15 controls fibroblast motility and wound closure by ERK1/2-dependent mechanisms. J Cell Physiol 227: 2106-2116, 2012.

11. Stassi G, Garofalo M, Zerilli M, Ricci-Vitiani L, Zanca C Todaro M, Aragona F, Limite G, Petrella G and Condorelli G: PED mediates AKT-dependent chemoresistance in human breast cancer cells. Cancer Res 65: 6668-6675, 2005.

12. Sun XP, Dong X, Lin L, Jiang X, Wei Z, Zhai B, Sun B, Zhang Q, Wang X, Jiang H, et al: Up-regulation of survivin by AKT and hypoxia-inducible factor $1 \alpha$ contributes to cisplatin resistance in gastric cancer. FEBS J 281: 115-128, 2014.

13. Trencia A, Perfetti A, Cassese A, Vigliotta G, Miele C, Oriente F, Santopietro S, Giacco F, Condorelli G, Formisano P, et al: Protein kinase B/Akt binds and phosphorylates PED/PEA-15, stabilizing its antiapoptotic action. Mol Cell Biol 23: 4511-4521, 2003.

14. Hayashi N, Peacock JW, Beraldi E, Zoubeidi A, Gleave ME and Ong CJ: Hsp27 silencing coordinately inhibits proliferation and promotes Fas-induced apoptosis by regulating the PEA-15 molecular switch. Cell Death Differ 19: 990-1002, 2012.

15. Li L, Jiang X, Zhang Q, Dong X, Gao Y, He Y, Qiao H, Xie F, $X$ ie $X$ and Sun $X$ : Neuropilin-1 is associated with clinicopathology of gastric cancer and contributes to cell proliferation and migration as multifunctional co-receptors. J Exp Clin Cancer Res 35: 16, 2016

16. Hu B, El Hajj N, Sittler S, Lammert N, Barnes R and Meloni-Ehrig A: Gastric cancer: Classification, histology and application of molecular pathology. J Gastrointest Oncol 3: 251-261, 2012.

17. Shin M, Lee KE, Yang EG, Jeon H and Song HK: PEA-15 facilitates EGFR dephosphorylation via ERK sequestration at increased ER-PM contacts in TNBC cells. FEBS Lett 589: 1033-1039, 2015

18. Livak KJ and Schmittgen TD: Analysis of relative gene expression data using real-time quantitative PCR and the $2^{-\Delta \Delta C_{\mathrm{T}}}$ method. Methods 25: 402-408, 2001.

19. Böck BC, Tagscherer KE, Fassl A, Krämer A, Oehme I, Zentgraf HW, Keith M and Roth W: The PEA-15 protein regulates autophagy via activation of JNK. J Biol Chem 285 21644-21654, 2010.

20. Wei Z, Jiang X, Qiao H, Zhai B, Zhang L, Zhang Q, Wu Y, Jiang $H$ and Sun X: STAT3 interacts with Skp2/p27/p21 pathway to regulate the motility and invasion of gastric cancer cells. Cell Signal 25: 931-938, 2013.

21. Ma L, Li G, Zhu H, Dong X, Zhao D, Jiang X, Li J, Qiao H, Ni S and Sun X: 2-Methoxyestradiol synergizes with sorafenib to suppress hepatocellular carcinoma by simultaneously dysregulating hypoxia-inducible factor-1 and -2. Cancer Lett 355: 96-105, 2014

22. Zhai B, Hu F, Jiang X, Xu J, Zhao D, Liu B, Pan S, Dong X, Tan G, Wei Z, et al: Inhibition of Akt reverses the acquired resistance to sorafenib by switching protective autophagy to autophagic cell death in hepatocellular carcinoma. Mol Cancer Ther 13: 1589-1598, 2014.

23. Han P, Li H, Jiang X, Zhai B, Tan G, Zhao D, Qiao H, Liu B Jiang $H$ and Sun X: Dual inhibition of Akt and c-Met as a second-line therapy following acquired resistance to sorafenib in hepatocellular carcinoma cells. Mol Oncol 11: 320-334, 2017.
24. Shah MA and Schwartz GK: Cell cycle-mediated drug resistance: An emerging concept in cancer therapy. Clin Cancer Res 7: 2168-2181, 2001.

25. Eckert A, Böck BC, Tagscherer KE, Haas TL, Grund K, Sykora J, Herold-Mende C, Ehemann V, Hollstein M, Chneiweiss H, et al: The PEA-15/PED protein protects glioblastoma cells from glucose deprivation-induced apoptosis via the ERK/MAP kinase pathway. Oncogene 27: 1155-1166, 2008.

26. Musgrove EA, Caldon CE, Barraclough J, Stone A and Sutherland RL: Cyclin D as a therapeutic target in cancer. Nat Rev Cancer 11: 558-572, 2011.

27. Stottrup C, Tsang T and Chin YR: Upregulation of AKT3 confers resistance to the AKT inhibitor MK2206 in breast cancer. Mol Cancer Ther 15: 1964-1974, 2016.

28. Hindupur SK, Balaji SA, Saxena M, Pandey S, Sravan GS, Heda N, Kumar MV, Mukherjee G, Dey D and Rangarajan A: Identification of a novel AMPK-PEA15 axis in the anoikis-resistant growth of mammary cells. Breast cancer Res 16: 420, 2014.

29. Zanca C, Garofalo M, Quintavalle C, Romano G, Acunzo M, Ragno P, Montuori N, Incoronato M, Tornillo L, Baumhoer D, et al: PED is overexpressed and mediates TRAIL resistance in human non-small cell lung cancer. J Cell Mol Med 12: 2416-2426, 2008.

30. Wang M, Zhu XY, Wang L and Lin Y: Expression and significance of CDC25B, PED/PEA-15 in esophageal carcinoma. Cancer Biother Radiopharm 30: 139-145, 2015.

31. Bartholomeusz C, Rosen D, Wei C, Kazansky A, Yamasaki F, Takahashi T, Itamochi H, Kondo S, Liu J and Ueno NT: PEA-15 induces autophagy in human ovarian cancer cells and is associated with prolonged overall survival. Cancer Res 68: 9302-9310, 2008.

32. Lee J, Bartholomeusz C, Krishnamurthy S, Liu P, Saso H, Lafortune TA, Hortobagyi GN and Ueno NT: PEA-15 unphosphorylated at both serine 104 and serine 116 inhibits ovarian cancer cell tumorigenicity and progression through blocking $\beta$-catenin. Oncogenesis 1: e22, 2012

33. Singh M, Chaudhry P, Fabi F and Asselin E: Cisplatin-induced caspase activation mediates PTEN cleavage in ovarian cancer cells: A potential mechanism of chemoresistance. BMC Cancer 13: 233, 2013.

34. Radisavljevic Z: AKT as locus of cancer multidrug resistance and fragility. J Cell Physiol 228: 671-674, 2013.

35. Li CW, Xia W, Lim SO, Hsu JL, Huo L, Wu Y, Li LY, Lai CC, Chang SS, Hsu YH, et al: AKT1 inhibits Epithelial-to-mesenchy mal transition in breast cancer through phosphorylation-dependent Twist1 degradation. Cancer Res 76: 1451-1462, 2016.

36. Serova M, de Gramont A, Tijeras-Raballand A, Dos Santos C, Riveiro ME, Slimane K, Faivre S and Raymond E: Benchmarking effects of mTOR, PI3K, and dual PI3K/mTOR inhibitors in hepatocellular and renal cell carcinoma models developing resistance to sunitinib and sorafenib. Cancer Chemother Pharmacol 71: 1297-1307, 2013.

37. Maiso P, Huynh D, Moschetta M, Sacco A, Aljawai Y, Mishima Y, Asara JM, Roccaro AM, Kimmelman AC and Ghobrial IM: Metabolic signature identifies novel targets for drug resistance in multiple myeloma. Cancer Res 75: 2071-2082, 2015. 